\title{
TENDÊNCIA DE MORTALIDADE POR CÂNCER EM MUNICÍPIO DO SUL DO BRASIL
}

\author{
Débora Cassiano Teixeira ${ }^{1}$ \\ Kátia Biagio Fontes ${ }^{2}$
}

TEIXEIRA, D. C.; FONTES, K. B. Tendência de mortalidade por câncer em município do sul do Brasil. Arq. Cienc. Saúde UNIPAR, Umuarama, v. 19, n. 2, p, 109-114, maio/ago. 2015.

RESUMO: Objetivou-se com este estudo avaliar a tendência da mortalidade por câncer no município de Umuarama, no período de 1987 a 2011. Foi realizado um estudo de série temporal utilizando-se dados demográficos e de mortalidade disponíveis na página da internet do Departamento de Informática do SUS (DATASUS). As taxas de mortalidade por câncer foram calculadas e agrupadas por quinquênios e calculado o percentual de variação. Os maiores percentuais de variação positiva nas taxas de mortalidade por câncer aconteceram entres as faixas etárias de 20 a 39 anos e 80 anos ou mais. Houve um percentual de variação negativa na maioria dos tipos de câncer, exceto no de mama e colón entre as mulheres, e próstata, entre os homens. O câncer de colo de útero foi o que exibiu um maior percentual de variação negativa entre as mulheres e o câncer de estômago entre os homens. Pode-se concluir que, entre os anos de 1987 a 2011, houve um aumento da tendência da mortalidade por câncer em ambos os gêneros nas faixas etárias entre 20 e 39 anos, e 80 anos. Entre as mulheres, houve um aumento da tendência da mortalidade por câncer de mama, seguido pelo de cólon e entre os homens, pelo câncer de próstata. O câncer de colo de útero entre as mulheres, seguido pelo câncer de estômago entre os homens, foi o que apresentou menor tendência à mortalidade no período estudado.

PALAVRAS-CHAVE: Coeficiente de mortalidade; Neoplasias; Registros de mortalidade.

\section{CANCER MORTALITY TRENDS IN THE CITY OF UMUARAMA, PARANÁ}

\begin{abstract}
This study aimed to evaluate the trend of cancer mortality in the municipality of Umuarama in the period from 1987 to 2011. A descriptive study was performed using demographic and mortality data available on the website of the SUS (Unified Health System) Department of Informatics (DATASUS). Mortality rates by cancer were calculated and grouped in a period of five years, calculating the variance percentage. The highest percentage of positive variance in cancer mortality rates occurred in the age groups from 20 to 39 years old and after 80 years old. There was a percentage of negative variance in most types of cancer, except for breast and colon cancers among women and prostate cancer among men. Cervical cancer was the one that exhibited the highest percentage of negative variance among women, and stomach cancer presented the highest percentage of negative variance among men. It can be concluded that between the years 1987 and 2011, the cancer mortality trend increased in both genders in the age groups between 20 and 39 years old, as well as in the 80-years-old age group. Among women, there was an increase in the breast cancer mortality trend, followed by colon cancer. Among men, the trend could be seen for prostate cancer. Cervical cancer in women, followed by stomach cancer in men showed the lowest mortality trends in the studied period.
\end{abstract}

KEYWORDS: Mortality Rate; Neoplasms; Mortality Registries.

\section{Introdução}

As Doenças Crônicas Não Transmissíveis (DCNT) constituem a principal causa de morte no mundo, sendo a maioria dos óbitos atribuíveis às doenças do aparelho circulatório, ao câncer, ao diabetes e às doenças respiratórias crônicas. No Brasil, essas contribuem por $72 \%$ das causas de morte, porém, na ultima década observou-se uma redução nas doenças no aparelho circulatório e respiratório, ao contrário das taxas de mortalidade por diabetes e câncer que aumentaram neste mesmo período (BRASIL, 2011).

De acordo com a Organização Mundial de Saúde (OMS), no ano de 2012, houve 14,1 milhões de novos casos e 8,2 milhões de mortes por câncer em todo o mundo. Para 2030 a estimativa de morte por câncer é de 13,2 milhões, dentre os 21,4 milhões de casos novos estimados (BRASIL, 2014a). No Brasil, dados do Ministério da Saúde (MS) indicam que entre os anos de 1996 a 2011, o câncer foi a segunda causa de morte ficando atrás apenas das doenças do aparelho cardiovascular (BRASIL, 2015a).

O Ministério da Saúde, em consonância com as diretrizes da Organização Mundial de Saúde (WORLD HEALTH ORGANIZATION, 2011), com o objetivo de "promover o desenvolvimento e a implementação de políticas públicas efetivas, integradas, sustentáveis e baseadas em evidências para a prevenção e o controle das DCNT e seus fatores de risco e fortalecer os serviços de saúde voltados para a atenção aos portadores de doenças crônicas", elaborou um Plano de Ações Estratégicas para o Enfrentamento das DCNT entre os anos de 2011-2022 (BRASIL, 2011, p.14).

Com relação às políticas de combate as DCNT no Brasil, especificamente as relacionadas ao câncer, tem-se destacado: o combate aos fatores de risco modificáveis (tabaco, atividade física insuficiente, uso nocivo do álcool, alimentação inadequada, excesso de peso e obesidade) e a ampliação de exames preventivos para os cânceres da mama e do colo do útero (BRASIL, 2011). Em 2014, o MS incorporou ao calendário vacinal do Sistema Único de Saúde a vacina contra o vírus do papiloma humano (HPV), usada na prevenção de câncer de colo do útero em pré-adolescentes de 11 a 13 anos, e em 2015 ampliou a faixa etária para meninas de 9 a 11 anos de idade (BRASIL, 2015b).

Diante do reconhecimento do câncer como problema de saúde pública, a influência desta neoplasia em termos epidemiológicos e a escassez de estudos de tendência de mortalidade por câncer no município de Umuarama, esse estudo teve como finalidade analisar a evolução da mortalidade por câncer no município, durante o período de 1987 a 2011.

DOI: https://doi.org/10.25110/arqsaude.v19i2.2015.5431

${ }^{1}$ Discente do Curso de Enfermagem da Universidade Paranaense - Unipar. E-mail: deeh_1@hotmail.com

${ }^{2}$ Docente do Curso de Enfermagem da Universidade Paranaense - Unipar. E-mail: katiabiagio@unipar.br 


\section{Material e Método}

Foi realizado um estudo de série temporal utilizando-se dados demográficos e de mortalidade para câncer de residentes em Umuarama, ocorridos entre 1987 a 2011. Os dados foram obtidos por meio do banco de dados do Sistema de Informações sobre Mortalidade (SIM) do Ministério da Saúde. As informações por gênero e faixa etária relativas à população residente de Umuarama foram obtidas no site do Departamento de Informática do Sistema Único de Saúde (Datasus) e têm como base os censos populacionais de 1980, 1991, 2000 e 2010, a recontagem de 1996 e as estimativas para os anos intercensitários da Fundação Instituto Brasileiro de Geografia e Estatística (IBGE).

Foram separados todos os óbitos de residentes de Umuarama, cuja causa básica foi o câncer, ocorridos no período de estudo, bem como para as seis principais localizações anatômicas de câncer em Umuarama, no mesmo período. Para o período de 1987-1995, foram conceituado como óbitos por neoplasia aqueles com causa básica codificados no capítulo II (Neoplasias), 9a da Classificação Estatística Internacional de Doenças e Problemas Relacionados à Saúde - $9^{\mathrm{a}}$ Revisão (CID-9) que engloba os códigos de 140.0 a 208. A partir de 1996, foram selecionados os óbitos com causa básica codificados no capítulo II da $10^{\mathrm{a}}$ Revisão da CID (CID-10: códigos de C00. 0 a C97).

As variáveis consideradas neste estudo foram gênero, idade, tipo específico de neoplasia e ano de ocorrência do óbito.

Os dados de mortalidade foram explorados em três quinquênios (1987-1991, 1997-2001 e 2007-2011) e associados nas seguintes faixas etárias: menores de 20 anos; 20-29 anos; 30-39 anos; 40-49 anos; 50-59 anos; 60-69 anos; 70-79 anos; e 80 e mais anos, com o objetivo de minimizar possí- veis oscilações aleatórias por conta da baixa frequência do evento estudado.

Para conferência dos dados, as taxas de mortalidade foram calculadas anualmente padronizadas por idade. Fez-se o cálculo do percentual de variação das taxas entre o primeiro e o último quinquênio mediante fórmula:

Taxa do quinquênio final - taxa do quinquênio inicial Taxa do quinquênio inicial

Para análise dos dados foi utilizado o software Excel 2007.

\section{Resultados}

Entre 1987 a 2011 foram registrados, em média, 80,5 casos de óbitos por câncer ao ano em Umuarama. As neoplasias de estômago, brônquios e pulmão, colón, próstata, mama e colo do útero, em conjunto, totalizam $45,21 \%$ de todas as mortes ocorridas por câncer nesse período.

Ao se analisar o período estudado, percebe-se que houve um crescente aumento das taxas de mortalidade ocasionando um percentual de variação positiva entre os quinquênios em ambos os gêneros. Entretanto, o maior percentual de variação apresentado foi entre as mulheres (Tabela 1).

Observa-se um crescente aumento das taxas de mortalidade a partir de 50 anos de idade, assim como decrescente nas demais faixas etárias. O grupo de 50 a 59 anos apresenta o maior percentual de variação negativo, sendo o grupo de 30 a 39 a maior variação positiva (Tabela 1). Aprecia-se em percentual de variação positiva das taxas de mortalidade por câncer nas mulheres entre 30 a 39 e 80 anos ou mais, já entre os homens este resultado se deu nas faixas etárias entre 20 a 39 anos e 80 anos e mais (Tabela 1).

Tabela 1: Taxas de mortalidade por câncer, por sexo, segundo faixa etária e percentual de variação no período de estudo. Umuarama, PR, Brasil, 1997 a 2011

\begin{tabular}{|c|c|c|c|c|c|c|c|c|c|c|c|c|}
\hline \multirow[b]{2}{*}{ Faixa etária } & \multicolumn{3}{|c|}{$\begin{array}{c}\text { Taxa de mortalidade }^{1} \\
\text { Gênero masculino }\end{array}$} & \multirow[t]{2}{*}{$\begin{array}{c}\text { Variação } \\
(\%)\end{array}$} & \multicolumn{3}{|c|}{$\begin{array}{c}\text { Taxa de mortalidade }{ }^{1} \\
\text { Gênero feminino }\end{array}$} & \multirow[t]{2}{*}{$\begin{array}{c}\text { Variação } \\
(\%)\end{array}$} & \multicolumn{3}{|c|}{$\begin{array}{c}\text { Taxas de mortalidade }{ }^{1} \\
\text { Ambos os Gêneros }\end{array}$} & \multirow[t]{2}{*}{$\begin{array}{c}\text { Variação } \\
(\%)\end{array}$} \\
\hline & 1987-91 & 1997-01 & 2007-11 & & 1987-91 & 1997-01 & 2007-11 & & $1987-91$ & 1997-01 & 2007-11 & \\
\hline$<20$ anos & 10,04 & 4,47 & 9,87 & $-1,69$ & 8,36 & 4,78 & 5,27 & $-36,96$ & 7,33 & 2,34 & 5,69 & $-22,37$ \\
\hline 20 a 29 anos & 6,69 & 10,64 & 15,89 & 137,51 & 10,33 & 4,94 & 4,47 & $-56,72$ & 8,58 & 7,68 & 10,14 & 18,18 \\
\hline 30 a 39 anos & 9,02 & 24,13 & 16,03 & 77,71 & 13,47 & 26,64 & 30,08 & 123,31 & 11,37 & 25,46 & 23,28 & 104,74 \\
\hline 40 a 49 anos & 67,37 & 60,69 & 60,39 & $-10,36$ & 97,11 & 88,42 & 66,64 & $-31,37$ & 82,09 & 75,49 & 63,71 & $-22,39$ \\
\hline 50 a 59 anos & 312,66 & 202,41 & 153,67 & $-50,85$ & 189,66 & 150,46 & 90,38 & $-52,34$ & 254,42 & 176,44 & 119,22 & $-53,14$ \\
\hline 60 a 69 anos & 469,23 & 478,47 & 392,55 & $-16,34$ & 343,33 & 263,43 & 238,31 & $-30,58$ & 410,42 & 370,65 & 311,82 & $-24,02$ \\
\hline 70 a 79 anos & 1288,66 & 1011,16 & 754,26 & $-41,46$ & 569,93 & 642,47 & 518,68 & $-8,99$ & 947,32 & 826,45 & 628,87 & $-33,6$ \\
\hline $\begin{array}{l}80 \text { anos e } \\
\text { mais }\end{array}$ & 1241,02 & 2472,95 & 1832,6 & 47,66 & 741,74 & 937,65 & 975,38 & 31,49 & 995,36 & 1669,12 & 1369 & 37,53 \\
\hline Total & 111,31 & 145,59 & 115,74 & 3,97 & 62,92 & 82,66 & 100,88 & 60,33 & 79,25 & 93,68 & 97,67 & 23,24 \\
\hline
\end{tabular}

${ }^{1}$ Taxas por 100.000 habitantes.

Ao analisar os tipos de câncer no período estudado, observou-se uma variação negativa na maioria dos tipos de câncer, exceto no de cólon, mama e próstata (Tabela 2). A mortalidade por tumores de cólon apresentou ascendência entre homens e mulheres nos primeiros dois quinquênios e declínio no terceiro quinquênio, porém, no gênero feminino, apresentou percentual de variação positiva entre os quinquê- nios (Tabela 2).

Nota-se uma curva decrescente das taxas de mortalidade por câncer de estômago e um percentual de variação negativa em todas as faixas etárias em todo o período estudado. Entre os tipos de câncer, o de estômago foi o que apresentou maior percentual de variação negativa (Tabela 2).

Apesar do câncer de estômago ter apresentado 
maior taxa de mortalidade entre homens e mulheres no primeiro quinquênio estudado, nos demais quinquênios, a maior taxa de mortalidade entre homens foi o câncer de próstata, e entre as mulheres, o câncer de mama (Tabela 2).

Em relação às taxas de mortalidade por câncer de próstata, houve variação positiva no período estudado, e o segundo quinquênio apresentou a maior taxa de mortalidade (Tabela 2). Com relação aos cânceres específicos das mulheres o de colo apresentou curva decrescente em todos os quinquênios, ao contrário do de mama que apresentou um elevado percentual de variação positivo. (Tabela 2). O câncer de colo de útero foi o que exibiu a maior percentual de variação negativa entre todos os tipos de câncer (Tabela 2).

Ao analisar as taxas de mortalidade por câncer de pulmão, houve um percentual de variação negativo em ambos os gêneros, contudo, as taxas de mortalidade entre os homens por câncer de pulmão foi maior em todos os quinquênios (Tabela 2).

Tabela 2: Taxas de mortalidade por localizações específicas de câncer, por sexo e na população em geral e percentual de variação no período de estudo. Umuarama, PR, Brasil, 1997 a 2011

\begin{tabular}{|c|c|c|c|c|c|c|c|c|c|c|c|c|}
\hline \multirow[b]{2}{*}{ Faixa etária } & \multicolumn{3}{|c|}{$\begin{array}{c}\text { Taxa de mortalidade }^{1} \\
\text { Gênero masculino }\end{array}$} & \multirow[t]{2}{*}{$\begin{array}{c}\text { Variação } \\
(\%)\end{array}$} & \multicolumn{3}{|c|}{$\begin{array}{c}\text { Taxa de mortalidade }^{1} \\
\text { Gênero feminino }\end{array}$} & \multirow[t]{2}{*}{$\begin{array}{c}\text { Variação } \\
(\%)\end{array}$} & \multicolumn{3}{|c|}{$\begin{array}{c}\text { Taxas de mortalidade } \\
\text { Ambos os Gêneros }\end{array}$} & \multirow[t]{2}{*}{$\begin{array}{c}\text { Variação } \\
(\%)\end{array}$} \\
\hline & 1987-91 & 1997-01 & 2007-11 & & 1987-91 & 1997-01 & 2007-11 & & 1987-91 & 1997-01 & 2007-11 & \\
\hline Estômago & 54,95 & 39,13 & 22,15 & $-59,69$ & 21,93 & 16,55 & 11,78 & $-46,28$ & 38,5 & 27,37 & 16,69 & $-56,64$ \\
\hline Cólon & 11,53 & 12,89 & 8,86 & $-23,15$ & 5,31 & 10,74 & 6,36 & 19,77 & 8,53 & 11,78 & 7,58 & $-11,13$ \\
\hline Pulmão & 26,53 & 39,13 & 22,97 & $-13,41$ & 9,54 & 19,46 & 8,84 & $-7,33$ & 18,06 & 28,89 & 15,52 & $-14,06$ \\
\hline Mama & - & - & - & - & 15,66 & 24,33 & 19,15 & 22,28 & - & - & - & - \\
\hline Colo de útero & - & - & - & - & 19,63 & 12,44 & 7,16 & $-63,52$ & - & - & - & - \\
\hline Próstata & 33,84 & 68,17 & 48,72 & 43,97 & - & - & - & - & - & - & - & - \\
\hline
\end{tabular}

${ }^{1}$ Taxas por 100.000 habitantes.

\section{Discussão}

Ao analisar a variação da taxa de mortalidade por câncer entre as faixas etárias separadamente, notam-se taxas em declínio do percentual de variação no grupo etário menor que 20 anos e entre 40 e 79 anos para ambos os gêneros, corroborando com estudo realizado nas capitais do Brasil entre os anos de 1980 a 2004, que revelou uma diminuição das taxas de mortalidade na faixa etária de 40 a 69 anos (FONSECA; ELUF-NETO; WUNSCH FILHO, 2010). Porém, após os 79 anos, as taxas de mortalidade por câncer se tornaram ascendentes em ambos os gêneros. Segundo o Instituto Brasileiro de Geografia e Estatística (IBGE), no estado do Paraná, a esperança de vida, ao nascer, para ambos os gêneros, no ano de 2014, foi 75,99 e a estimativa é de que, em 2030, chegará a 79,35 anos. O acesso à população aos serviços de saúde, prevenção de doenças, campanhas de vacinação, avanços da tecnologia são alguns dos fatores que contribuíram para a prevenção secundária e tratamento, consequentemente a sobrevida e qualidade de vida aumentaram, o que se traduz na redução da mortalidade (BRASIL, 2010a).

Quando são analisados os tipos de câncer mais prevalentes no município, observa-se que o câncer de estômago e pulmão exibiram um declínio em todos os quinquênios estudados, tanto no gênero masculino como no feminino. Porém, ao analisar o câncer de pulmão por quinquênio observa-se que o declínio da taxa se deu somente a partir do segundo quinquênio. Tal dado se assemelha ao resultado de estudo anterior realizado no Brasil, onde esse tipo de câncer apresentou ascendência entre os anos de 1980 e 2006 (SILVA et al., 2011). Estudo, nas capitais brasileiras, entre os anos de 2006 a 2009 revelaram uma leve tendência da diminuição do uso de tabaco entre homens e estabilidade entre as mulheres (SILVA; VALENTE; MALTA, 2011). Tais resultados sugerem que, nos últimos anos, a adoção de hábitos saudáveis, entre eles, a diminuição do uso do tabaco pela população de homens e mulheres do município pode ter influenciado a queda na taxa de mortalidade.

O câncer de estômago se mostrou descendente em todos os grupos conforme resultado de estudo anterior (SILVA et al., 2011). Segundo o INCA, as melhorias no saneamento básico, a utilização de alimentos conservados em refrigeração, em vez do uso do sal e defumação para tal finalidade, além de uma dieta equilibrada composta por vegetais crus, frutas cítricas e alimentos ricos em fibras, o combate do tabaco e redução ingesta de bebidas alcoólicas são medidas que resultam na prevenção contra câncer de estômago (BRASIL, 2014a).

Por outro lado, o câncer de cólon no gênero feminino demonstrou variação positiva no período estudado, corroborando com resultados de estudos que analisaram as taxas de mortalidade por câncer de colo no Brasil entre os anos de 1980 a 2004 (FONSECA; ELUF-NETO; WUNSCH FILHO, 2010) e estado de Santa Catarina de 1980 a 2006 (VASQUES; PERES, 2010). No Brasil os programas de detecção para o câncer de colo retal são raros e limitados pelo sistema de saúde. Ao considerar a alta incidência da doença, o diagnóstico precoce é de suma importância. A pesquisa sobre a presença de sangue oculto nas fezes com questionários de sinais e sintomas mostra-se um método efetivo de prevenção para o rastreamento do câncer colo retal (ALTENBURG; SIMÕES; BAHTEN, 2009).

Nos cânceres específicos das mulheres, houve redução do câncer de colo conforme resultado de estudo desenvolvido no Brasil entre os anos de 1980 e 2006 (SILVA et al., 2011), porém, aumento do câncer de mama. Resultado de outro estudo realizado no estado de Goiás evidenciou uma estabilidade na taxa de mortalidade por câncer de colo e uma significativa redução desse câncer, após a implantação do Programa Viva Mulher no estado, houve uma diminuição da mudança percentual anual, refletindo eficiência das ações de prevenção, diagnóstico e tratamento (SANTANA; REZENDE; MANRIQUE, 2013). Recentemente o MS incorporou a vacina contra o HPV ao calendário nacional . A vacina contra 
HPV disponível pelo Ministério da Saúde tem como finalidade a prevenção primária do câncer de colo de útero, e com esse avanço na ciência, espera-se um impacto na redução do câncer de colo de útero de 30 a 40 anos. Com a vacinação consequentemente haverá uma redução na carga de doença cervical e lesões precursoras (BRASIL, 2010b).

Apesar do rastreamento do câncer de mama no país pelo sistema de saúde, sua incidência pode estar relacionada ao aumento da expectativa de vida da população e, portanto, maior prevalência de seus fatores de risco (FONSECA; ELUF-NETO; WUNSCH FILHO, 2010). Por outro lado, apesar de menos incidente em mulheres mais jovens, estudo revelou que o aumento da mortalidade por câncer de mama no Brasil não é homogêneo e vem destacando-se nas mulheres com até 50 anos (MARTINS et al., 2013). Resultado de estudo realizado em Santa Catarina evidenciou que, entre as mulheres, a faixa etária com maior risco de óbito foi a abaixo de 30 anos, com um risco 4,15 vezes maior que as mulheres com mais de 70 anos (SCHNEIDER; ORSI, 2009).

Recentemente, no país, o Ministério da Saúde por meio da Portaria 1.253/2013, reduziu o direito do acesso a mulheres abaixo de 50 anos ao exame de mamografia pelo SUS como forma de rastreamento (BRASIL, 2013), assegurado anteriormente pela Lei $11.664 / 08$ a todas as mulheres a partir dos 40 anos de idade (BRASIL, 2008). De acordo com nota do INCA, para a faixa etária abaixo dos 50 anos, o Ministério da Saúde cobre a mamografia unilateral, sem rastreamento populacional, em qualquer faixa etária, desde que a paciente apresente sintomas ou histórico de câncer na família conforme solicitação médica. Estudos científicos têm demonstrado maior eficácia do exame de rastreamento feito a cada dois anos para as mulheres com 50 a 69 anos de idade. A recomendação por priorizar tal faixa etária é feita pela Organização Mundial da Saúde e seguida por diversos países que, há décadas, mantêm programas organizados de rastreamento do câncer mamário. Estudos indicam que a partir dos 50 anos, o tecido mamário é substituído pela gordura e, por isso, a observação de um possível tumor ou calcificação maligna se torna mais clara. A mamografia abaixo dos 50 anos não tem o mesmo resultado, quando é realizada na faixa prioritária (BRASIL, 2014b). Atualmente o Ministério da Saúde recomenda que o rastreamento de câncer de mama bianual por meio de mamografia para mulheres seja entre 50 e 74 anos (BRASIL, 2009).

O câncer de próstata foi único câncer entre os homens que mostrou uma variação positiva. Estudos anteriores também demonstraram aumento da taxa de mortalidade por câncer de próstata no Brasil entre os anos de 1980 e 2006 (SILVA et al., 2011) e nas capitais do Brasil entre os anos de 1980 e 2004 (FONSECA; ELUF-NETO; WUNSCH FILHO, 2010)

De acordo com a Organização Mundial da Saúde (OMS) a detecção precoce de um câncer envolve o diagnóstico precoce das pessoas que apresentam sinais iniciais da doença e o rastreamento de pessoas aparentemente saudáveis. O rastreamento do câncer de próstata é realizado por meio da realização do toque retal e dosagem de Antígeno Prostático específico (PSA) (BRASIL, 2015b). Contudo, para o Ministério da Saúde, devido a incertezas em torno do teste, não é recomendada organização de ações de rastreamento devido a resultados falso-positivos, infecções e sangramentos resultantes de biópsia. Soma-se isto a ansiedade agregada ao diagnostico do câncer e tratamento de casos que não iriam evoluir clinicamente (BRASIL, 2010c). Não há evidências suficientes para compor as recomendações a favor ou contra a adesão do rastreamento para o câncer de próstata em homens que não apresentem sintomas com idade inferior aos 75 anos, pois sua incidência é rara antes dos 50 anos, e o risco aumenta com a idade. Homens que solicitem a realização do exame de rastreamento devem ser informados por seus médicos sobre os riscos e benefícios associados a essa prática e posteriormente devem definir-se em conjunto com a equipe de saúde pela realização ou não do rastreamento. Diante da posição de não realização pelo $\mathrm{MS}$, o mesmo recomenda a capacitação dos profissionais de saúde para a identificação precoce dos casos por meio do conhecimentos dos sinais e sintomas de alerta, o pronto acesso dos casos suspeitos à investigação diagnóstica. No caso de confirmação diagnóstica o paciente deve ser encaminhado imediatamente para tratamento, nos casos de solicitação de exames de rastreamento deve-se esclarecer os possíveis riscos e benefícios relacionados aos exames e sobre os sinais e sintomas suspeitos. Não realizar campanhas para convocar homens assintomáticos para a realização de rastreamento com PSA e/ou toque retal (BRASIL, 2014c). A Lei 17.099/2002 institui o mês "agosto azul" para desenvolvimentos de atividades voltadas a integralidade da saúde do homem, realizando ações preventivas e campanhas de esclarecimento priorizando assuntos como cardiologia, urologia (câncer de próstata), saúde mental e pneumologia (BRASIL, 2012).

\section{Conclusão}

Pode-se concluir que entre os anos de 1987 e 2011, houve um aumento da tendência da mortalidade por câncer somente nas faixas etárias dos 20 aos 39, e 80 anos ou mais em ambos os gêneros, porém, ao se analisar por sexo, a faixa etária entre 20 e 29 anos apresentou aumento apenas entre os homens.

Todos os tipos de câncer apresentaram variação negativa, porém, quando analisado por sexo, o de próstata apresentou-se aumentado nos homens, e entre as mulheres o de mama seguido pelo de cólon. $\mathrm{O}$ câncer de colo de útero entre as mulheres, seguido pelo câncer de estômago entre os homens, foram os que apresentaram menor tendência à mortalidade no período estudado.

\section{Referências}

ALTENBURG, F. L.; SIMÕES, M. L. P. B.; BAHTEN, L. C. V. A pesquisa de sangue oculto nas fezes associada a um questionário de sinais e sintomas na prevenção do câncer colo retal. Revista Brasileira Coloproctologia, v. 29, n. 1, 2009. Disponível em: < http://www.scielo.br/pdf/rbc/v29n1/ v29n1a08.pdf > . Acesso em: 05 maio 2015.

BRASIL. Ministério da Saúde. Instituto Nacional de Câncer José Alencar Gomes da Silva. Coordenação de Prevenção e Vigilância. Estimativa 2014. Incidência de Câncer no Brasil. Instituto Nacional de Câncer José Alencar Gomes da Silva, Coordenação de Prevenção e Vigilância. Rio de Janeiro: INCA, 2014. 124 p. Disponível em: <http://www. 
inca.gov.br/estimativa/2014/estimativa-24042014.pdf>. ideiaAcesso em: 05 maio 2015.

.ideiaPortal da saúde. Vacinação contra o HPV - 2015. Disponível em: <http://portalarquivos.saude. gov.br/campanhas/hpv/>.ideiaAcesso em: 05 maio 2015.

Secretaria de Vigilância em Saúde.

Departamento de Análise de Situação de Saúde. Plano de ações estratégicas para o enfrentamento das doenças crônicas não transmissíveis (DCNT) no Brasil 2011-2022 / Ministério da Saúde. Secretaria de Vigilância em Saúde. Departamento de Análise de Situação de Saúde. - Brasília: Ministério da Saúde, 2011. 160 p.

Secretaria de Vigilância em Saúde. Departamento de Análise da Situação de Saúde. Sistema de informações sobre mortalidade (SIM). Brasília: Ministério da Saúde, 2015. Disponível em: <http://www. datasus.gov.br>. Acesso em: 05 maio 2015.

Secretaria de Atenção à Saúde. Departamento de Ações Programáticas e Estratégicas. Atenção à saúde da pessoa idosa e envelhecimento. Ministério da Saúde, Secretaria de Atenção à Saúde, Departamento de Ações Programáticas e Estratégicas, Área Técnica Saúde do Idoso. Brasília, 2010a. Disponível em: <http://bvsms.saude. gov.br/bvs/publicacoes/atencao_saude_pessoa_idosa envelhecimento_v12.pdf>.ideiaAcesso em: 05 maio 2015.

Instituto Nacional de Câncer. Plano de ação para redução da incidência e mortalidade por câncer do colo do útero: sumário executivo/ Instituto Nacional de Câncer. Rio de Janeiro: INCA, 2010. Disponível em: $<$ http://bvsms.saude.gov.br/bvs/publicacoes/plano_acao_ reducao_cancer_colo.pdf $>$. Acesso em: 05 maio 2015.

Instituto Nacional do Câncer. Ministério da Saúde. Acesso à informação: agencia de noticias. Noticias: Posicionamento do Ministério da Saúde sobre a realização de exames de mamografia no SUS, 2014b. Disponível em: <http://www2.inca.gov.br/wps/wcm/connect/ agencianoticias/site/home/noticias/2014/posicionamentoministerio_saude_sobre_realizacao_exames_mamografia sus $>$. Acesso em: 05 maio 2015.

Instituto Nacional de Câncer. Parâmetros para o rastreamento do câncer de mama: recomendações para gestores estaduais e municipais. Instituto Nacional de Câncer. Rio de Janeiro: INCA, 2009. $13 \mathrm{p}$.

Instituto Nacional de Câncer José Alencar Gomes da Silva. Próstata: detecção precoce. Disponível em: <http://www2.inca.gov.br/wps/wcm/connect/ tiposdecancer/site/home/prostata/deteccao_precoce $>$. Acesso em: 05 maio 2015.

Secretaria de Atenção à Saúde.

Departamento de Atenção Básica. Rastreamento.

Ministério da Saúde, Secretaria de Atenção à Saúde,
Departamento de Atenção Básica. - Brasília: Ministério da Saúde, 2010c. Disponível em: <http://bvsms.saude. gov.br/bvs/publicacoes/caderno_atencao_primaria_29 rastreamento.pdf $>$. Acesso em: 05 maio 2015.

Instituto Nacional de Câncer José Alencar

Gomes da Silva. Informativo: detecção precoce.

Monitoramento das ações de controle do câncer de próstata.

Boletim, a. 5,ideian. 2 maio/ ago. 2014. Disponível em:

$<$ http://www1.inca.gov.br/inca/Arquivos/Informativo_

Deteccao_Precoce_2_agosto_2014.pdf $>$.ideiaAcesso em: 05 maio 2015 .

Secretaria de Atenção à Saúde. Portaria no

1.253, de 12 de novembro de 2013. Altera atributos de procedimentos na Tabela de Procedimentos, Medicamentos, Órteses, Próteses e Materiais Especiais do Sistema Único de Saúde. A Secretária de Atenção à Saúde - Substituta, no uso de suas atribuições, 2013. Disponível em: $<$ http://bvsms. saude.gov.br/bvs/saudelegis/sas/2013/prt1253_12_11_2013. html>. Acesso em: 05 maio 2015.

Presidência da República. Casa Civil. Subchefia para Assuntos Jurídicos. Lei no 11.664, de 29 de abril de 2008. Dispõe sobre a efetivação de ações de saúde que assegurem a prevenção, a detecção, o tratamento e o seguimento dos cânceres do colo uterino e de mama, no âmbito do Sistema Único de Saúde, SUS. Presidência da República Brasília, 29 de abril de 2008. Disponível em: $<$ http://www.planalto.gov. br/ccivil_03/_ato2007-2010/2008/lei/111664.htm>. Acesso em: 05 maio 2015.

\section{Casa Civil. Subchefia para Assuntos}

Jurídicos. Lei no 17.099, de 28 de março de 2012. Institui o mês "Agosto Azul", dedicado ao desenvolvimento de ações que visem à integralidade da saúde do homem. Presidência da República Brasília, 28 de março de 2012. Disponível em: $<$ http://www.legislacao.pr.gov.br/legislacao/listarAtosAno. do ?action $=$ exibir\&codAto $=66181 \&$ indice $=1 \& \operatorname{anoSpan}=201$ $2 \&$ anoSelecionado $=2012 \&$ mesSelecionado $=0 \&$ isPaginado $=$ true $>$ Acesso em: 05 maio 2015 .

FONSECA, L. A.; ELUF-NETO, J.; WUNSCH FILHO, V. Tendências da mortalidade por câncer nas capitais dos estados do Brasil, 1980-2004. Rev Assoc Med Bras. v. 56 n. 3, 2010. Disponível em: <http://www.scielo.br/pdf/ramb/ v56n3/v56n3a15.pdf>. Acesso em: 05 maio 2015.

MARTINS, C. A. et al. Evolução da mortalidade por câncer de mama em mulheres jovens: desafios para uma política de atenção oncológica. Revista Brasileira de Cancerologia, v. 59, n. 3, 2013. Disponível em: <http://www.inca.gov. $\mathrm{br} / \mathrm{rbc} / \mathrm{n}$ _59/v03/pdf/04-artigo-evolucao-mortalidadecancer-mama-mulheres-jovens-desafios-politica-atencaooncologica.pdf $>$. Acesso em: 05 maio 2015.

SANTANA, C. K. L. S. L.; REZENDE, S. R. F; MANRIQUE, E. J. C. Tendência de mortalidade por câncer do colo do útero no estado de Goiás no período de 1989 a 2009. Revista Brasileira de Cancerologia, v. 59 n. 1, 2013. Disponível em: <http://www.inca.gov.br/rbc/n_59/ 
v01/pdf/03-tendencia-de-mortalidade-por-cancer-do-colodo-utero-no-estado.pdf>. Acesso em: 05 maio 2015.

SCHNEIDER, I. J. C.; ORSI, E. Sobrevida em cinco anos e fatores prognósticos em mulheres com câncer de mama em Santa Catarina, Brasil. Cad. Saúde Pública, v. 25, n. 6, 2009. Disponível em: <http://www.scielo.br/pdf/csp/ v25n6/11.pdf $>$. Acesso em: 05 maio 2015.

SILVA, G. A. et al. Tendência da mortalidade por câncer nas capitais e interior do Brasil entre 1980 e 2006. Rev Saúde Pública, v. 45, n. 6, 2011. Disponível em: <http://www. scielo.br/pdf/rsp/v45n6/2963.pdf > . Acesso em: 05 maio 2015.

SILVA, G. A.; VALENTE, J. G.; MALTA, D. C. Tendências do tabagismo na população adulta das capitais brasileiras: uma análise dos dados de inquéritos telefônicos de 2006 a 2009. Revista Brasileira Epidemiologia, v. 14, n. 1, 2011. Disponível em: <http://www.scielo.br/pdf/rbepid/v14s1/ a11v14s1.pdf $>$. Acesso em: 05 maio 2014.

VASQUES, A. L.; PERES, M. A. Tendência temporal da mortalidade por câncer de cólon e reto em Santa Catarina no período entre 1980 a 2006. Epidemiol. Serv. Saúde, v. 19, n. 2, 2010. Disponível em: <http://www.dive.sc.gov. br/conteudos/publicacoes/livros_artigos/Ana_Luiza Epidemiologia_Servicos_Saude_2010.pdf $>$. Acesso em: 05 maio 2015.

\section{WORLD HEALTH ORGANIZATION. Global status} report on noncommunicable diseases 2010 . Geneva: WHO, 2011. 164 p. Disponível em: <http://www.who.int/ $\mathrm{nmh} /$ publications/ncd_report2010/en>. Acesso em: 05 maio 2015. 